\title{
The Role of the BDNF Val66Met Polymorphism for the Synchronization of Error-Specific Neural Networks
}

\author{
Christian Beste, ${ }^{1}$ Vasil Kolev, ${ }^{3}$ Juliana Yordanova, ${ }^{3}$ Katharina Domschke, ${ }^{2}$ Michael Falkenstein, ${ }^{4}$ Bernhard T. Baune, ${ }^{5}$ \\ and Carsten Konrad $2,6,7$ \\ ${ }^{1}$ Institute for Cognitive Neuroscience, Department of Biopsychology, Ruhr-Universität Bochum, D-44780 Bochum, Germany, ${ }^{2}$ Department of Psychiatry \\ and Psychotherapy, University of Münster, D-48149 Münster, Germany, ${ }^{3}$ Institute of Neurobiology, Bulgarian Academy of Sciences, Sofia, 1113 Sofia, \\ Bulgaria, ${ }^{4}$ IFADO-Leibniz Institute, Dortmund, D-44139 Dortmund, Germany, ${ }^{5}$ Department of Psychiatry and Psychiatric Neuroscience, James Cook \\ University, Townsville, Queensland, Australia, ${ }^{6}$ Interdisciplinary Center for Clinical Research (IZKF), University of Münster, D-48129 Münster, Germany, \\ and ${ }^{7}$ Department of Psychiatry and Psychotherapy, University of Marburg, D-35037 Marburg, Germany
}

Behavioral adaptation depends on the recognition of response errors and processing of this error-information. Error processing is a specific cognitive function crucial for behavioral adaptation. Neurophysiologically, these processes are reflected by an event-related potential (ERP), the error negativity (Ne/ERN). Even though synchronization processes are important in information processing, its role and neurobiological foundation in behavioral adaptation are not understood. The brain-derived neurotrophic factor (BDNF) strongly modulates the establishment of neural connectivity that determines neural network dynamics and synchronization properties. Therefore altered synchronization processes may constitute a mechanism via which BDNF affects processes of error-induced behavioral adaptation. We investigate how variants of the BDNF gene regulate EEG-synchronization processes underlying error processing. Subjects $(n=$ 65) were genotyped for the functional BDNF Val66Met polymorphism (rs6265). We show that Val/Val genotype is associated with stronger error-specific phase-locking, compared with Met allele carriers. Posterror behavioral adaptation seems to be strongly dependent on these phase-locking processes and efficacy of EEG-phase-locking-behavioral coupling was genotype dependent. After correct responses, neurophysiological processes were not modulated by the polymorphism, underlining that BDNF becomes especially necessary in situations requiring behavioral adaptation. The results suggest that alterations in neural synchronization processes modulated by the genetic variants of BDNF Val66Met may be the mechanism by which cognitive functions are affected.

\section{Introduction}

The brain-derived neurotrophic factor (BDNF) influences differentiation and survival of neurons, synaptic plasticity and connectivity in the brain (Cohen-Cory et al., 2010). A single-nucleotide polymorphism in the human BDNF gene, resulting in a valine to methionine substitution in the prodomain (Val66Met), has been shown to influence cognitive performance in normal conditions and susceptibility to neuropsychiatric disorders that are related to cognitive dysfunctions (for review, see Bath and Lee, 2006). The BDNF Val66Met polymorphism alters the secretion of the mature peptide (Egan et al., 2003). Compared with the Met allele, the Val allele is associated with higher activity of the BDNF system (Rybakowski, 2008). Notably, the Val allele also is associated with an enhancement, whereas the Met allele is linked to a diminution of cognitive functions (Kleim et al., 2006; Goldberg et al.,

\footnotetext{
Received May 16, 2010; accepted June 21, 2010.

The study was supported by a young investigator grant to C.K. by the Interdisciplinary Centre for Clinical Research of the University of Münster, Germany (IZKF FG4), partly by a grant from the Ruhr-University of Bochum, FoRUM AZ F647-2009 and the Deutschte Forschungsgemeinschaft (BE 4045/8-1) to C.B., and by the National Council for Scientific Research at the Ministry of Education and Science, Sofia, Bulgaria (Project L-1501/2005 to J.Y.).

Correspondence should be addressed to Christian Beste, Institute for Cognitive Neuroscience, Department of Biopsychology, Ruhr-Universität Bochum, Universitätsstraße 150, D-44780 Bochum, Germany. E-mail: christian. beste@rub.de.

DOI:10.1523/JNEUROSCI.2493-10.2010

Copyright $\odot 2010$ the authors $\quad 0270-6474 / 10 / 3010727-07 \$ 15.00 / 0$
}

2008), although opposite effects have been reported (Beste et al., 2010b). Although BDNF is relevant for cognitive abilities and maintenance of functional neural circuits, it is not clear how the BDNF-mediated effects on cognitive processes emerge (Bath and Lee, 2006). Possibilities include largely distributed changes in structural (Cohen-Cory et al., 2010) and functional connectivity (Thomason et al., 2009). As connectivity determines neural network synchronization and dynamics (Lago-Fernández et al., 2001; Kitano and Fukai, 2007), synchronization may constitute a relevant mechanism by which BDNF affects cognitive functions. Such possible mechanisms have until now not been investigated.

Here, we examine how the functional BDNF Val66Met polymorphism (rs6265) modulates synchronization mechanisms supporting error monitoring and behavioral posterror readjustment that play a key role for higher cognitive functioning (Falkenstein et al., 2000). Error processing is reflected by a specific electroencephalographic (EEG) signal in the brain, the error negativity (Ne/ERN) (Falkenstein et al., 1991; Gehring et al., 1993) that is observed as a component of the response-related potentials (RRPs) in the brain. Concerning Ne/ERN generation, it is suggested that if an event is worse than expected (i.e., an error), the basal ganglia send an error-signal via the dopaminergic system to the anterior cingulate (ACC). The ACC in turn elicits the Ne/ERN (Holroyd and Coles, 2002) and triggers subsequent behavioral adjustments (Debener et al., 2005). Relative 
to correct responses, error generation is accompanied by stronger neural synchronization mechanisms associated with intensified performance monitoring and behavioral adaptation (Yordanova et al., 2004b; Kolev et al., 2009).

Basing on the differential effects of functional BDNF Val66Met polymorphism on cognitive functions (Kleim et al., 2006; Goldberg et al., 2008) we hypothesize that Val allele carriers show an enhanced error monitoring with elevated posterror adaptation, relative to Met allele carriers. These differences would be reflected by a larger Ne/ERN (indicating increased error monitoring) and by a pronounced posterror slowing [indicating improved posterror adaptation (Rabbitt, 1966)] in Val/Val relative to $\mathrm{Val} / \mathrm{Met}$ or Met/Met carriers. With regard to the important role of BDNF for the establishment of inter-regional connectivity, which affects synchronizing properties of neural networks, it may be further hypothesized that increased phase-synchrony underlies enhanced error monitoring in Val allele carriers.

\section{Materials and Methods}

\section{Subjects}

A sample of 65 genetically unrelated, healthy subjects of Caucasian descent was recruited by newspaper announcements. Genotyping of the BDNF Val66Met polymorphism (see below) showed that 30 subjects carried the $\mathrm{Val} / \mathrm{Val}$ genotype, 25 carried the $\mathrm{Val} / \mathrm{Met}$ genotype and 10 carried the Met/Met genotype. Hardy-Weinberg equilibrium was examined using the program Finetti provided as an online source (http://ihg.gsf.de/cgi-bin/hw/hwal.pl; T. F. Wienker and T. M. Strom). The distribution of BDNF Val66Met genotypes did not significantly differ from the expected numbers calculated on the basis of observed allele frequencies according to Hardy-Weinberg equilibrium ( $p=0.225)$. As the Met/Met genotype had an expectedly low frequency, we combined $\mathrm{Val} / \mathrm{Met}$ and Met/Met genotypes to one genotype group. Means and SDs are provided to describe demographical data. The sample consisted of 30 males and 35 females with mean age of 25.4 ( \pm 4.9$)$ years. Gender was equally distributed across genotype groups [Kruskal-Wallis test $(\mathrm{H}-$ Test): $\left.\chi^{2}=0.04 ; \mathrm{df}=1 ; p>0.7\right]$. The Beck Depression Inventory (BDI) score was $4.1( \pm 3.6)$ and did not differ between the genotype groups $\left(F_{(1,63)}=0.3 ; p>0.6\right)$. Similarly, the anxiety sensitivity index (ASI mean/SD: $11.4 \pm 3.4)$ did not differ between genotype groups $\left(F_{(1,63)}=\right.$ $0.6 ; p>0.3)$. Volunteers were paid 8 Euros per hour as compensation. The study was approved by the ethics committee of the University of Münster. All subjects gave written informed consent before any of the study procedures were commenced.

\section{Genotyping}

Genotyping of the genetic variants of BDNF Val66Met SNP rs6265 (position: chr11:27,636,492) was performed following published protocols applying the multiplex genotyping assay iPLEX for use with the MassARRAY platform (Oeth et al., 2007), yielding a genotyping completion rate of $97.7 \%$. Genotypes were determined by investigators blinded to the study.

\section{Experimental paradigm}

Flanker tasks are often used to examine error-related processes (Beste et al., 2008, 2010b). These tasks are well suited for this purpose because of the possibility to increase error rates especially in incompatible trials. Compatible and incompatible trials are created by means of flankers (triangles pointing to the left or right) that precede a centrally presented target stimulus. The experimental paradigm and analytic procedures are illustrated in Figure 1.

The stimulus onset asynchrony (SOA) was set to $100 \mathrm{~ms}$ to maximize premature responding to the flankers, i.e., flanker stimuli were presented $100 \mathrm{~ms}$ before the onset of the target. Flankers and target were switched off simultaneously. Target stimuli were also triangles pointing to the left or right. Subjects were asked to respond with the left or right thumb, depending on the direction of the target triangle (left vs right). The configuration of the flankers and targets provokes errors especially in the incompatible condition, where arrowheads of flankers and the target

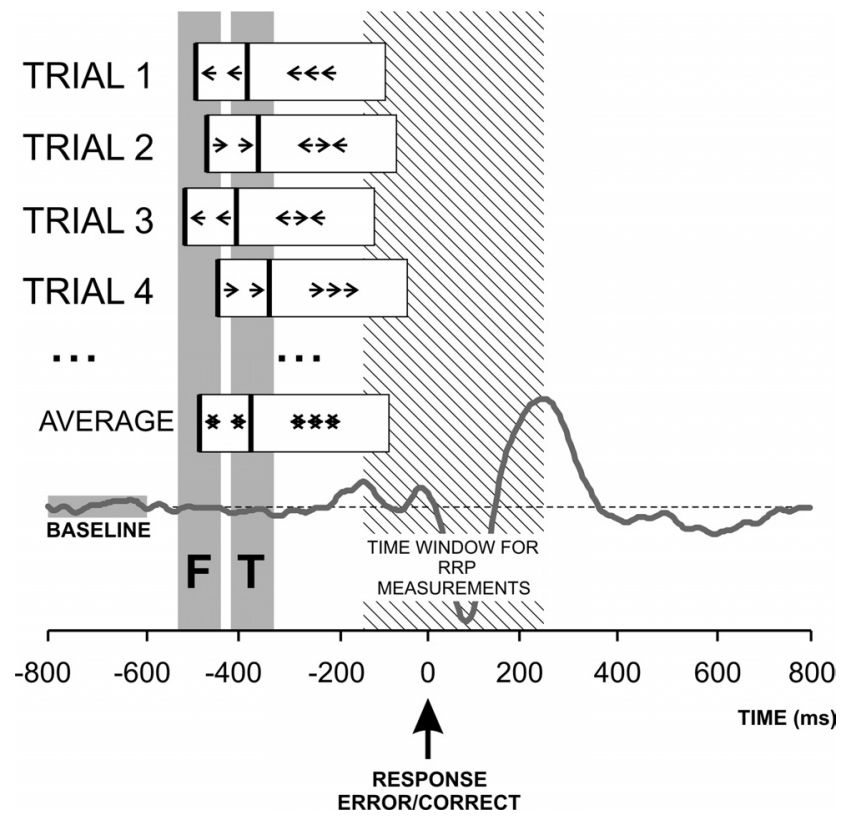

Figure 1. Schematic diagram of task stimuli. Examples of the timing of four single-trial stimuli are shown (trial 1 to trial 4 ) together with their average. The range of flanker (F) and target (T) onset variations (gray bars) is presented relative to response onset $(0 \mathrm{~ms})$ to help understand how RRPs were quantified. F and T are separated by a SOA equal to $100 \mathrm{~ms}$. The hatched box denotes the time window for RRP amplitude, total power, and phase-locking measurements. The baseline was always set from $800-600$ ms before response production. In the stimulus boxes, examples for flanker and target arrows are illustrated. The participant is instructed to respond with the respective hand according to the direction of the middle target arrow.

point in opposite directions. The target stimulus was displayed for 300 ms. The response-stimulus interval was $1600 \mathrm{~ms}$, a new trial was delivered once $1600 \mathrm{~ms}$ after the response in the previous trial was made. This interval was jittered between 1300 and $1600 \mathrm{~ms}$ to avoid expectancy effects related to the flanker. Time pressure was administered by asking the subjects to respond within $600 \mathrm{~ms}$, which further increases the likelihood of response errors. In trials with reaction times exceeding this deadline a feedback stimulus $(1000 \mathrm{~Hz}, 60 \mathrm{~dB}$ SPL) was given $1200 \mathrm{~ms}$ after the response; this stimulus had to be avoided by the subjects.

\section{Behavioral analysis}

The number of correct and error trials was measured to reflect differences in performance accuracy. Reaction times (RT) to correct and error responses were recorded and subjected to statistical analysis. An index of error processing is posterror slowing, which is a slowing in RTs when an error has occurred (Rabbitt, 1966). To calculate this posterror slowing, we measured the mean RT of correct responses after correct and after error reactions, which formed a within-subject factor "sequence" in a repeated-measures ANOVA with a between-subject factor "genotype group."

\section{EEG data processing}

EEG signals were recorded from $28 \mathrm{Ag}-\mathrm{AgCl}$ electrodes (Fpz, Fp1, Fp2, Fz, F3, F4, F7, F8, FCz, FC3, FC4, FC5, FC6, Cz, C3, C4, C7, C8, Pz, P3, $\mathrm{P} 4, \mathrm{P} 7, \mathrm{P} 8, \mathrm{Oz}, \mathrm{O} 1, \mathrm{O}_{2}$, left and right mastoids-M1/M2) against a reference electrode located on Cz. Additionally, eye movements were monitored and recorded by means of two lateral and four vertical EOG electrodes. The sampling rate of all recordings was 500 samples/s, applying a filter bandwidth of $0.05-80 \mathrm{~Hz}$ to the EEG. Electrode impedances were kept below $5 \mathrm{k} \Omega$. Artifact rejection procedures were applied twice: automatically, with an amplitude threshold of $\pm 80 \mu \mathrm{V}$, and visually by rejecting all trials contaminated by technical artifacts. Horizontal and vertical eye movements preserved in the accepted trials were corrected by means of a linear regression method for EOG correction (Gratton et al., 1983). Before quantifying RRPs, the current source density (CSD) of the 
signals was calculated to achieve a reference-free evaluation (Perrin et al., 1989; Nunez et al., 1997). The exact mathematical procedure is explained in detail in the work by Perrin et al. (1989). In the present study, the following parameters were used: order of splines $\mathrm{m}=4$, and the maximum degree of the Legendre polynomials $n=10$, with a precision of $2.72^{-7}$.

To achieve a reliable analysis of slow frequency components in subsequent time-frequency (TF) analyses, the EEG was segmented into 4096 ms long epochs with the response (error or correct) production starting in the center of the analysis epoch (time point 0 ). A preresponse baseline was defined for the time window -800 until $-600 \mathrm{~ms}$ before button press, which, according to the reaction times, represents a time window from the prestimulus period and it is free of activity related to stimulus or response processing. All subsequent peak quantification was done relative to this baseline. Figure 1 depicts the time course of stimuli and reactions in relation to the RRP quantification procedures.

\section{Time domain analysis}

To evaluate general characteristics of error processing, error and correct averaged RRPs were analyzed in the time domain. On error trials, the $\mathrm{Ne} / \mathrm{ERN}$ was defined as the most negative peak and measured within a time window of 50 until $110 \mathrm{~ms}$ postresponse (Falkenstein et al., 2000). The time domain Ne/ERN was measured peak-to-peak against the preceding positivity. On correct trials, the correct response negativity $\mathrm{Nc} /$ CRN was defined and measured following the same criteria. For quantification of RRPs in both the time and time-frequency domains, only trials were used for which RTs were between 150 and $900 \mathrm{~ms}$. Trials falling out of this interval were discarded from analysis.

\section{Time-frequency decomposition}

TF analysis of RRPs was performed by means of a continuous wavelet transform (CWT) (Mallat, 1999) with Morlet wavelets as basis functions. Here, the TF energy during response execution was analyzed by means of a modification of a method described previously (Tallon-Baudry et al., 1997). Complex Morlet wavelets $w$ can be generated in the time domain for different frequencies, $f$, according to the equation: $w(t, f)=$ $A \exp \left(-t^{2} / 2 \sigma_{t}^{2}\right) \exp (2 i \pi f t)$, where $t$ is time, $A=\left(\sigma_{t} \sqrt{\pi}\right)^{-1 / 2}, \sigma_{t}$ is the wavelet duration, and $i=\sqrt{-1}$. For analysis and TF-plots, a ratio of $f_{0} / \sigma f=5.5$ was used, where $f_{0}$ is the central frequency and $\sigma_{f}$ is the width of the Gaussian shape in the frequency domain. The choice of the ratio $f_{0} / \sigma_{f}$ was oriented to the expected slower phase-locked components present in the RRPs, which had an effect on the shape of the Morlet wavelet and decreased its decay (for more explanations, see Yordanova et al., 2004b). The analysis was performed in the frequency range $0.1-16 \mathrm{~Hz}$ with a central frequency at $0.4 \mathrm{~Hz}$ intervals. For different $f_{0}$, time and frequency resolutions can be calculated as $2 \sigma_{t}$ and $2 \sigma_{f}$, respectively. $\sigma_{t}$ and $\sigma_{f}$ are related by the equation $\sigma_{t}=1 /\left(2 \pi \sigma_{f}\right)$. For example, for $f_{0}=3$ $\mathrm{Hz}, 2 \sigma_{t}=425 \mathrm{~ms}$, and $2 \sigma_{f}=1.5 \mathrm{~Hz}$; for $f_{0}=5 \mathrm{~Hz}, 2 \sigma_{t}=255 \mathrm{~ms}$ and $2 \sigma_{f}=2.5 \mathrm{~Hz}$.

TF analysis was performed for both averaged and single-trial RRPs. TF decomposition of averaged RRPs was done to identify the frequency and time localization of TF $\mathrm{Ne} / \mathrm{Nc}$ subcomponents that were most strongly synchronized during error/correct response processing with the assumption that these phase-locked subcomponents are emphasized by averaging, whereas the non-phase-locked ones are attenuated. Relevant frequency bands were detected with central frequencies $f_{0}$ of $1.56,3.02$, and $4.84 \mathrm{~Hz}$ (see Results). TF decomposition was also performed at the level of nonaveraged single trials. Single-trial analysis aimed at assessing separately the magnitude and phase-synchronization of TF subcomponents. Accordingly, only single-trial measures were subjected to statistical evaluation.

Total power. To analyze the magnitude of TF components, we obtained the TF power for each single response and then averaged across trials. This approach quantifies the total activity (total power) comprising the phase-locked and non-phase-locked fractions of the responserelated activity (for more theoretical explanations, see Kalcher and Pfurtscheller, 1995). For each trial, the time-varying power in a given frequency band was calculated, which was obtained by squaring the absolute value of the convolution of the signal with the complex wavelet.
Frequency-relevant TF power was extracted in relevant frequency ranges, and analyzed. The maximal TF power and the corresponding peakpower latencies were measured within the time window $150 \mathrm{~ms}$ before and $250 \mathrm{~ms}$ after the response production (Fig. 1). A time window of $600-800 \mathrm{~ms}$ before the response was used to estimate background activity. The mean of this baseline epoch was subtracted from the TF power measures at each time point of the analysis epoch for each frequency band and electrode. For statistical analysis, TF power was $\log 10$ transformed to normalize the distributions.

Phase-locking. Neural synchronization mechanisms can be quantified at the level of single-trial EEG data by using the phase-locking factor (PLF) (Tallon-Baudry et al., 1997, 2001; Roach and Mathalon, 2008; Kolev et al., 2009). The PLF provides a measure of the synchronization of oscillatory activity independently of the signal's amplitude (Kolev and Yordanova, 1997; Yordanova and Kolev, 1998). The values of PLF yield a value between 0 and 1 determining the degree of phase-locking, where 1 indicates perfect phase alignment across trials and values close to 0 reflect the highest phase variability.

\section{Statistical analysis}

Kolmogorov-Smirnov tests revealed that all relevant variables were normally distributed (all $z<0.7 ; p>0.3$; one-tailed). Data were analyzed using repeated and univariate analyses of variance (ANOVAs). In the repeated-measures ANOVAs, the factors "electrode (2 levels: Fz, FCz)" and "correctness (2 levels: error vs correct)" were used as within-subject factors, "BDNF genotype group (2 levels: Val/Val vs Val/Met-Met/Met)" was used as a between-subject factor. When appropriate, the degrees of freedom were adjusted using Greenhouse-Geisser correction. Further, if necessary, the performed post hoc tests were corrected according to the Bonferroni procedure. As a measure of variability, the SEM together with the mean values are given. All statistical analyses were computed by using the software package SPSS 15.0.

\section{Results}

\section{Behavioral data}

\section{Reaction times}

RTs were faster on errors ( $325 \pm 8 \mathrm{~ms})$ than on correct trials $\left(383 \pm 9 \mathrm{~ms} ;\left(F_{(1,61)}=90.62 ; p<0.001 ; \eta=0.590\right)\right.$. This effect was not different across genotype groups as indicated by the nonsignificant interaction $\left(F_{(1,61)}=0.12 ; p>0.7 ; \eta=0.002\right)$. The main effect of genotype was also nonsignificant $\left(F_{(1,61)}=0.1 ; p>\right.$ $0.9 ; \eta=0.001)$.

\section{Error rates}

While error rates were higher in incompatible than in compatible conditions $\left(F_{(1,61)}=180.97 ; p<0.001 ; \eta=0.733\right)$, there were no interactions with genotype $\left(F_{(1,61)}=1.34 ; p>0.2\right)$. Also, the mean error rate did not differ between the genotype groups $\left(F_{(1,61)}=0.45 ; p>0.5 ; \eta=0.007\right)$.

\section{Posterror slowing}

There was a general slowing effect after errors $\left(F_{(1,61)}=192.54\right.$; $p<0.001 ; \eta=0.753)$ showing that RTs after errors were longer $(396 \pm 10 \mathrm{~ms})$ than after correct trials in succession $(371 \pm 8)$. Notably, the degree of slowing was different for the genotype groups, as indicated by the interaction $\left(F_{(1,61)}=59.74 ; p<0.001\right.$; $\eta=0.487)$. Post hoc tests revealed that posterror slowing was stronger in the Val/Val genotype group ( $34 \pm 4 \mathrm{~ms})$, compared with the combined Val/Met-Met/Met genotype group $(11 \pm 3)$ $\left(F_{(1,61)}=21.56 ; p<0.001 ; \eta=0.255\right)$.

\section{Time domain RRPs}

Grand average waveforms at electrode $\mathrm{FCz}$ including scalp topography are illustrated in Figure $2 \mathrm{~A}$.

The repeated-measures ANOVA revealed an expected main effect "correctness" $\left(F_{(1,61)}=150.56 ; p<0.001 ; \eta=0.723\right)$, with negative amplitudes being larger on error compared with correct 

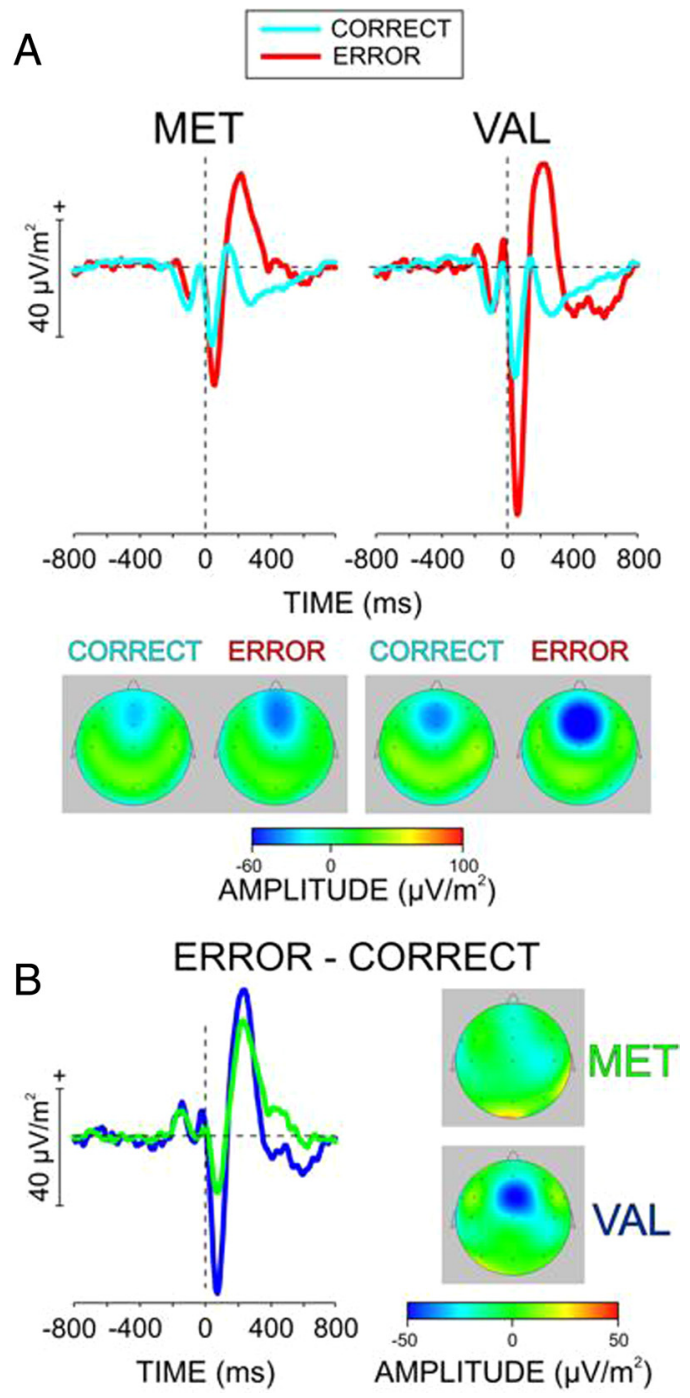

Figure 2. Grand average response-related potentials for error and correct trials at electrode FCz and their difference, as well as the corresponding topography maps at the maximal peaks of error and correct response-related negativities. Time 0 ms denotes the point of response production. $\boldsymbol{A}$, Response-related potentials obtained from the error and correct trials for the combined Val/Met-Met/Met (MET) and for the Val/Val (VAL) genotype group. The maps shown on the bottom present the typical frontal/frontal-central topography for the error and correct response-related potentials. $\boldsymbol{B}$, Response-related difference waves (error minus correct) and the corresponding topography maps for the Val/Met-Met/Met (MET) and Val/Val (VAL) genotype groups.

trials. As shown in the figure and indicated by the interaction "correctness $\times$ genotype group" $\left(F_{(1,61)}=63.61 ; p<0.001 ; \eta=\right.$ $0.502)$, this effect was different across the genotype groups. As demonstrated in Figure $2 B$ and indicated by subsequent univariate ANOVAs, the Val/Val group had a larger Ne/ERN $(-60.1 \pm$ $1.8 \mu \mathrm{V} / \mathrm{m}^{2}$ ) than the combined $\mathrm{Val} /$ Met-Met/Met group $\left(-35.2 \pm 1.7 \mu \mathrm{V} / \mathrm{m}^{2}\right)\left(F_{(1,61)}=94.92 ; p<0.001 ; \eta=0.601\right)$. No genotype differences were obtained for correct trials $\left(F_{(1,61)}=\right.$ $1.48 ; p>0.2 ; \eta=0.023)$. RRP amplitudes were overall larger in the $\mathrm{Val} / \mathrm{Val}\left(-42.0 \pm 1.3 \mu \mathrm{V} / \mathrm{m}^{2}\right)$ than in the combined $\mathrm{Val} /$ Met-Met/Met group $\left(-28.5 \pm 1.2 \mu \mathrm{V} / \mathrm{m}^{2}\right)\left(F_{(1,61)}=65.19 ; p<\right.$ $0.001 ; \eta=0.509)$

There was an interaction "electrode $\times$ correctness $\times$ genotype group" $\left(F_{(1,61)}=27.63 ; p<0.001 ; \eta=0.509\right)$. Post hoc tests indicated that this interaction was due to a larger genotype effects at electrode $\mathrm{FCz}(t=-13.92 ; \mathrm{df}=29 ; p<0.001)$, compared with
$\mathrm{Fz}(t=-9.37 ; \mathrm{df}=29 ; p<0.001$; see also Fig. $2 B)$. No significant electrode differences across genotype groups were obtained for amplitudes of correct trials. All other main or interaction effects were not significant (all F's $<1 ; p>0.4$ ). Although the latency of the $\mathrm{Ne} / \mathrm{ERN}(74 \pm 2 \mathrm{~ms}$ ) was longer than for the $\mathrm{Nc}(48 \pm 2 \mathrm{~ms})$ $\left(F_{(1,61)}=154.40 ; p<0.001 ; \eta=0.710\right)$, this difference was not modulated by the genotype (for all other effects and interactions F's $<0.5 ; p>0.6$ ).

Overall, the $\mathrm{Val} / \mathrm{Val}$ genotype group revealed a stronger $\mathrm{Ne}$ and concomitantly stronger behavioral adaptation compared with the Val/Met-Met/Met genotype group, suggesting enhanced error monitoring and behavioral adaptation processes.

\section{Time-frequency RRPs}

Since genotype effects between error and correct trials were largest at electrode $\mathrm{FCz}$ (Fig. $2 \mathrm{~B}$ ), we used this electrode for all TF analyses. Figure $3 A$ illustrates TF plots of averaged RRPs to correct and error responses, and difference RRPs in the Val/Val and $\mathrm{Val} / \mathrm{Met}-\mathrm{Met} / \mathrm{Met}$ groups at FCz.

It is demonstrated that correct trials in the two genotype groups were basically characterized by phase-locked TF components in the $\theta(4-8 \mathrm{~Hz})$ band. In contrast, error trials contained a major $\delta(2-4 \mathrm{~Hz})$ component that was virtually absent on correct trials. These observations are consistent with previously described TF components of RRPs induced by correct and error trial processing (Yordanova et al., 2004b). The major $\delta$-component can best be seen in the difference wavelet plots. However, in these plots also a sub- $\delta$ component was evident in the Met allele group.

Following the observations of TF components of averaged RRPs described above, $\delta$ and $\theta$ TF components were included in single-trial analyses. The sub- $\delta$ band was omitted from the present analysis because it might not be specifically associated with errors and also because of its larger time scale where other activities induced by stimulus processing or variations in RT due to trial compatibility/incompatibility or accuracy might be overlapped (Yordanova et al., 2004b). Frequency band ( $\delta$ and $\theta$ ) was included as a within-subjects factor in the analysis to assess whether differences between genotype groups were linked to specific TF components or reflected a general property of the genotype. Therefore, only interactive effects of frequency band with genotype group will be regarded in the following.

Total power. Figure $3 A$ illustrates TF-plots of RRP total power to correct and error responses, and difference plots in the $\mathrm{Val} / \mathrm{Val}$ and Val/Met-Met/Met groups at FCz. A significant interaction "frequency band $\times$ genotype group" $\left(F_{(1,63)}=33.75 ; p<0.001\right.$; $\eta>0.349)$ indicated that group differences in total power existed, but they were differentially expressed for the different frequency bands. Subsequent univariate ANOVAs revealed no group differences in the $\theta$-frequency band $\left(F_{(1,63)}=0.53 ; p>0.4\right.$; $\eta=0.008)$. The groups only differed in the $\delta$-frequency band (Val/Val: $4.33 \pm 0.02 ; \mathrm{Val} / \mathrm{Met}-\mathrm{Met} / \mathrm{Met}: 4.12 \pm 0.01 ; F_{(1,63)}=$ $72.68 ; p<0.001 ; \eta=0.536)$. This indicates that especially errorspecific TF components are differentially modulated the BDNF Val66Met polymorphism.

Phase-locking. Figure $3 B$ shows TF plots of the PLF to correct and error responses, and difference TF plots for the $\mathrm{Val} / \mathrm{Val}$ and $\mathrm{Val} / \mathrm{Met}-\mathrm{Met} / \mathrm{Met}$ groups at FCz. Phase-locking was higher in the $\delta$ than $\theta$ frequency band $\left(F_{(1,63)}=43.34 ; p<0.001 ; \eta=0.408\right)$. Moreover, PLF was higher on error than on correct trials $\left(F_{(1,63)}=\right.$ 182.54; $p<0.001 ; \eta=0.753)$. Of note, there was an interaction "frequency band $\times$ correctness $\times$ genotype group" $\left(F_{(1,63)}=15.19\right.$; $p=0.001 ; \eta=0.194)$. Separate repeated-measures ANOVAs for 

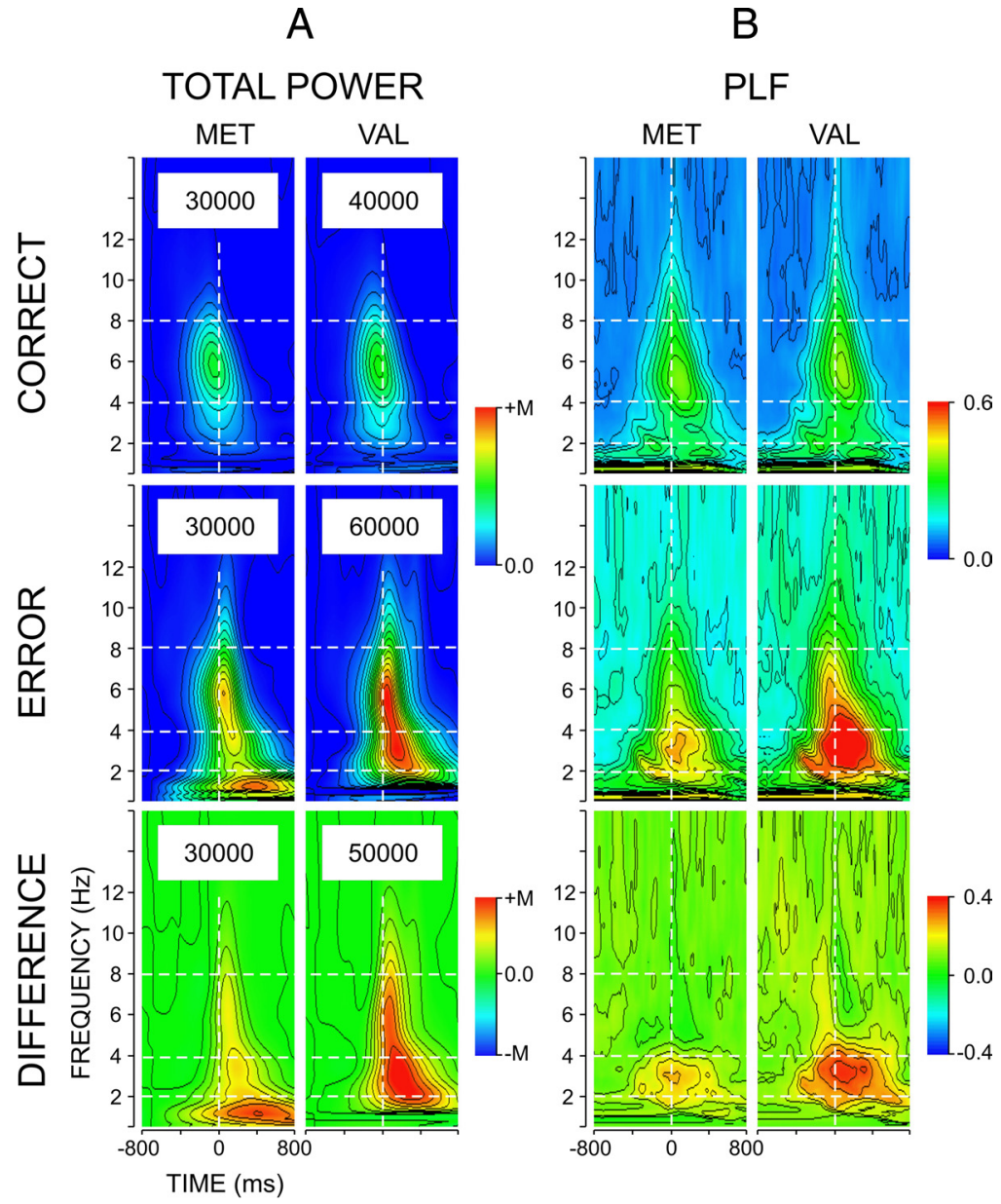

Figure 3. Grand average time-frequency plots for correct, error, and difference (error minus correct) response-related potentials for both genotype groups: Val/Met-Met/Met (MET) and Val/Val (VAL). A, Grand average total power. Horizontal dashed lines denote the boundaries of the obtained significant time-frequency components (sub- $\delta, \delta$, and $\theta$ ). Vertical dashed lines at $0 \mathrm{~ms}$ denote the point of response production. The numbers in the white boxes show the calibration power values $\mathrm{M}\left(\mu \mathrm{V}^{2} / \mathrm{m}^{4}\right)$ for each plot. $\boldsymbol{B}$, Grand average PLF. PLF values vary between 0 and 1 for all plots.

error and correct trials across genotype groups revealed that this effect was driven by the error trials (correct trials: all F's $<0.90$; $p>0.7$; error trials: all F's $>7.12 ; p<$ $0.010)$. Hence, only error trials were analyzed further.

On error trials, the $\mathrm{Val} / \mathrm{Val}$ group revealed a stronger phase-locking (0.53 \pm 0.02) than the combined Val/Met-Met/Met genotype group $(0.47 \pm 0.03)$. However the interaction "frequency band $\times$ genotype group" $\left(F_{(1,63)}=10.84 ; p<0.001 ; \eta=\right.$ 0.147 ) indicated that this effect depended on the frequency of TF components. Subsequent post hoc tests revealed that only in the $\delta$ frequency band was the phase-locking stronger for the $\mathrm{Val} / \mathrm{Val}$ than for the combined Val/Met-Met/Met genotype group $\left(F_{(1,63)}=47.41 ; p<0.001 ; \eta=0.429\right)$.

\section{Regression analyses}

The reported results suggest that phaselocked wavelet power and the PLF on error trials show genotype-dependent variations, especially in the $\delta$-frequency band. At a behavioral level, posterror slowing showed genotype-dependent variations. Posterror slowing was stronger in the $\mathrm{Val} / \mathrm{Val}$ genotype group, which also displayed higher phase-locked power and stronger PLF.

We conducted a linear regression analysis using the degree of posterror slowing as a dependent variable. The phase-locking within the sub- $\delta, \delta$ and $\theta$ frequency bands as well as the factor "genotype group" served as predictors that were step wisely entered into the model. The model shows that posterror slowing was best predicted by the phase-locking in the $\delta$ frequency band and genotype $\left(F_{(2,64)}=154.1 ; p<\right.$ 0.001) ( $\delta$ phase-locking: $t=10.43$; $p<$

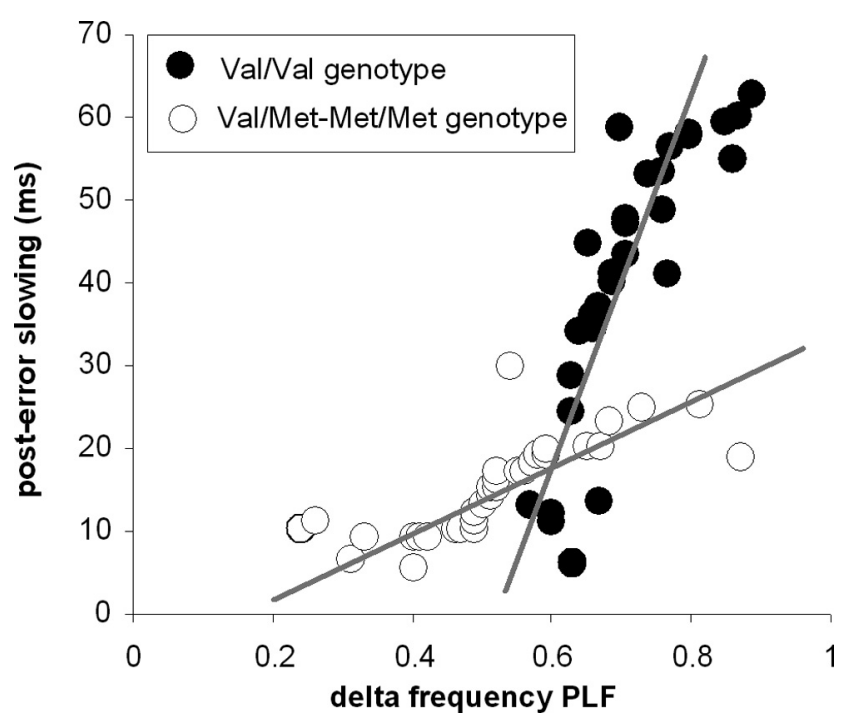

Figure 4. Scatter plot of posterror slowing vs phase-locking factor values measured in the $\delta$ frequency range for the $\mathrm{Val} / \mathrm{Val}$ and $\mathrm{Val} / \mathrm{Met}-\mathrm{Met} / \mathrm{Met}$ genotype group. Regression lines are presented for each group. Note the different slopes of the regression lines.
0.001 ; genotype: $t=-3.82$; $p<0.001)$. The relation between phase-locking in the $\delta$ frequency band and the degree of posterror slowing for each genotype group is illustrated in Figure 4.

Figure 4 suggests that the slopes of the regression lines were different for the $\mathrm{Val} / \mathrm{Val}$ and combined $\mathrm{Val} / \mathrm{Met}-\mathrm{Met} / \mathrm{Met}$ genotype group. Subsequent regression analyses for each genotype group separately underlined this. The regression coefficient was 154.5 for the Val/Val genotype group and 43.6 for the Val/MetMet/Met genotype group. This indicates that changes in $\delta$ phaselocking cause stronger effects in posterror slowing in the $\mathrm{Val} / \mathrm{Val}$ genotype group, compared with the Val/Met-Met/Met genotype group. The correlation between posterror slowing and phaselocking in the $\delta$ frequency band was similar for both groups (Val/ Val: $r=0.744 ; R^{2}=0.55 ; p<0.001 ; \mathrm{Val} /$ Met-Met/Met: $r=$ $\left.0.739 ; R^{2}=0.54 ; p<0.001\right)$. A similar regression analysis using the total power did not reveal a significant model.

\section{Discussion}

The influence of genetic factors on error processing and response monitoring functions has frequently been studied in the past (Klein et al., 2007; Krämer et al., 2007; Beste et al., 2010a). Until now, no study examined the role of neurotrophins for these im- 
portant cognitive functions. Furthermore, a fine-meshed analysis in terms of differences in neural synchronization processes that are important for information processing (Varela et al., 2001) and that may underlie observed genotype-dependent differences has not been carried out. To fill this gap, we examined modulatory influences of the functional BDNF Val66Met polymorphism (rs6265) on the neuronal dynamics underlying error processing, with special emphasizes on neural synchronization processes.

We show that the Val/Val genotype has an increased Ne/ERN, compared with the combined Val/Met-Met/Met genotype group. This pattern is mirrored by the behavioral data showing stronger posterror slowing effects in the $\mathrm{Val} / \mathrm{Val}$ compared with the $\mathrm{Val} /$ Met-Met/Met genotype group. Notably, potentials on correct trials did not differ between the groups, underlining that the results obtained are specific for error-related processes. In the $\mathrm{Val} / \mathrm{Val}$ group, increased synchronization was the leading marker for error-specific processing, while in the Val/Met-Met/Met group, increase in the total power at slower frequency components distinguished error processing.

In relation to further genotype differences in error processing, the synchronization analysis revealed that $\delta$-frequency Ne/ERN component was more strongly synchronized in the $\mathrm{Val} / \mathrm{Val}$ than in the combined Val/Met-Met/Met genotype group. No differences in synchronization were seen for other frequency bands or in the correct trials. This relative enhancement in the Ne/ERN in the $\mathrm{Val} / \mathrm{Val}$ genotype compared with the $\mathrm{Val} / \mathrm{Met}-\mathrm{Met} / \mathrm{Met}$ genotype group is most likely due to an increased $\delta$-frequency band total power and increased phase-locking within this frequency band. From a perspective of network functioning, the increase in error-specific total power suggests that error-specific neural populations are increasingly activated upon error-response generation. The concomitant increase in error-specific phase-synchronization (PLF) in the Val/Val genotype group further suggests that these populations also become more synchronized. Since motor responses are generated during both correct and error trials, the stronger synchronization of $\delta$ networks by error responses may not be associated with the mere motor aspect of response production (Yordanova et al., 2004b). If differences in the kinematics of the motor response (dependent on the different genotypes) produce different characteristics of the negative RRPs in the first 100 $\mathrm{ms}$ after response production, such effects would be present also in the CRN. The present results give clear evidence that the CRN in the time domain, as well as CRN TF subcomponents do not differentiate between the two genotype groups, suggesting that the results are unbiased with respect to motor generation processes.

The current results confirm previous observations that error responses specifically increase $\delta$-frequency band activity (Yordanova et al., 2004b; Beste et al., 2007, 2009, 2010a; Kolev et al., 2009). The results suggest also that BDNF gene variation might selectively modulate error-specific processes, but not processes of general motor response monitoring, since genotype effects were restricted to the $\delta$-frequency band, which was predominantly active on error, but not on correct trials (Yordanova et al., 2004b). This underlines that BDNF becomes especially important in situations requiring behavioral adaptation. The BDNF Val66Met polymorphism may insofar contribute to differences in neural synchronization processes and constitute an important element in determining how error-related information is processed. The degree of synchronization in the $\delta$-frequency band was related to the degree of behavioral adaptation after an error, suggesting that especially genotype differences in synchronization in this frequency band drive differentially efficient behavioral adaptation.
The regression coefficients show that the strength of association between $\delta$ frequency phase-locking and posterror slowing is stronger in the $\mathrm{Val} / \mathrm{Val}$ than in the $\mathrm{Val} / \mathrm{Met}-\mathrm{Met} / \mathrm{Met}$ genotype group. This implies that the $\mathrm{Val} / \mathrm{Val}$ genotypes may be related to a more efficient neural network that enables a stronger neuralbehavioral coupling subserved primarily by synchronization rather than power network properties (cf. Debener et al., 2005; Cavanagh et al., 2009).

The above described synchronization effects may be explained in the following way. As far as BDNF influences structural neuroanatomy (Pezawas et al., 2004), the arborization of dendritic spines (Cohen-Cory et al., 2010) and hence the connectivity of neural networks, the neural networks in Met-allele carriers may not be able to synchronize to an extent as observed in the Val/Val genotype group. This may cause differences in cognitive abilities, in particular abilities promoting behavioral adaptation. Related to these micro-structural reasons, differences between genotype groups may be further augmented by reductions in synaptic efficacy (Foltynie et al., 2009; Kleim et al., 2006) and/or dopaminergic neural transmission in Met allele carriers. The dopamine system may be of special relevance, because of its important role in error processing (Beste et al., 2006; Jocham and Ullsperger, 2008 ) and known interactions with BDNF on a receptor level (Do et al., 2007; Iwakura et al., 2008).

In summary, the present study suggests that the BDNF Val66Met polymorphism (rs6265) modulates error processing functions and subsequent processes of behavioral adaptation. The results suggest that alterations in neural synchronization processes modulated by the BDNF Val66Met variant may be the mechanism by which cognitive functions are affected. Similar mechanisms may underlie the genotype effects in other cognitive functions, which may be a target of future research. Moreover, the present results provide further evidences for error-specific processes in the brain that are distinguishable from other performance monitoring subprocesses on a neurobiological level. It is likely that this dissociation emerges as a consequence of different neural populations underlying error-specific and general motor monitoring functions.

\section{References}

Bath KG, Lee FS (2006) Variant BDNF (Val66Met) impact on brain structures and function. Cogn Affect Behav Neurosci 6:79-85.

Beste C, Saft C, Andrich J, Gold R, Falkenstein M (2006) Error processing in Huntington's disease. PloS One 1:e86.

Beste C, Saft C, Yordanova J, Andrich J, Gold R, Falkenstein M, Kolev V (2007) Functional compensation or pathology in cortico-subcortical interactions in preclinical Huntington's disease. Neuropsychologia 45:2922-2930.

Beste C, Saft C, Konrad C, Andrich J, Habbel A, Schepers I, Jansen A, Pfleiderer B, Falkenstein M (2008) Levels of error processing in Huntington's disease: a combined study using event-related potentials and voxel-based morphometry. Hum Brain Mapp 29:121-130.

Beste C, Willemssen R, Saft C, Falkenstein M (2009) Error processing in normal aging and in basal ganglia disorders. Neuroscience 159:143-149.

Beste C, Domschke K, Kolev V, Yordanova J, Baffa A, Falkenstein M, Konrad C (2010a) Functional 5-HT1a receptor polymorphism selectively modulates error-specific subprocesses of performance monitoring. Hum Brain Mapp 31:621-630.

Beste C, Baune BT, Domschke K, Falkenstein M, Konrad C (2010b) Paradoxical association of the brain-derived-neurotrophic-factor val66met genotype with response inhibition. Neuroscience 166:178-184.

Cavanagh JF, Cohen MX, Allen JJ (2009) Prelude to and resolution of an error: EEG phase synchrony reveals cognitive control dynamics during action monitoring. J Neurosci 29:98-105.

Cohen-Cory S, Kidane AH, Shirkey NJ, Marshak S (2010) Brain-derived neurotrophic factor and the development of structural neuronal connectivity. Dev Neurobiol 70:271-288. 
Debener S, Ullsperger M, Siegel M, Fiehler K, von Cramon DY, Engel AK (2005) Trial-by-trial coupling of concurrent electroencephalogram and functional magnetic resonance imaging identifies the dynamics of performance monitoring. J Neurosci 25:11730-11737.

Do T, Kerr B, Kuzhikandathil EV (2007) Brain-derived neurotrophic factor regulates the expression of D1 dopamine receptors. J Neurochem 100:416-428.

Egan MF, Kojima M, Callicott JH, Goldberg TE, Kolachana BS, Bertolino A, Zaitsev E, Gold B, Goldman D, Dean M, Lu B, Weinberger DR (2003) The BDNF val66met polymorphism affects activity-dependent secretion of BDNF and human memory and hippocampal function. Cell 112:257-269.

Falkenstein M, Hohnsbein J, Hoormann J, Blanke L (1991) Effects of crossmodal divided attention on the ERP components. II. Error processing in choice reaction tasks. Electroencephalogr Clin Neurophysiol 78:447-455.

Falkenstein M, Hoormann J, Christ S, Hohnsbein J (2000) ERP components on reaction error and their functional significance: a tutorial. Biol Psychol 51:87-107.

Foltynie T, Cheeran B, Williams-Gray CH, Edwards MJ, Lewis SG, Schneider SA, Weinberger D, Rothwell JC, Barker RA, Bhatia KP (2009) BDNF val66met influences time to onset of levodopa induced dyskinesia in Parkinson's disease. J Neurol Neurosurg Psychiatry 80:141-144.

Gehring WJ, Goss B, Coles MGH, Meyer DE, Donchin E (1993) A neural system for error detection and compensation. Psychol Sci 4:385-390.

Goldberg TE, Iudicello J, Russo C, Elvevåg B, Straub R, Egan MF, Weinberger DR (2008) , BDNF Val66Met polymorphism significantly affects $\mathrm{d}^{\prime}$ in verbal recognition memory at short and long delays. Biol Psychol 77:20-24.

Gratton G, Coles MG, Donchin E (1983) A new method for off-line removal of ocular artefact. Electroencephalogr Clin Neurophysiol 55:468-484.

Holroyd CB, Coles MG (2002) The neural basis of human error processing: reinforcement learning, dopamine, and the error-related negativity. Psychol Rev 109:679-709.

Iwakura Y, Nawa H, Sora I, Chao MV (2008) Dopamine D1 receptorinduced signalling through TrkB Receptors in striatal neurons. J Biol Chem 283:15799-15806.

Jocham G, Ullsperger M (2008) Neuropharmacology of performance monitoring. Neurosci Biobehav Rev 3:48-60.

Kalcher J, Pfurtscheller G (1995) Discrimination between phase-locked and non-phase-locked event-related EEG activity. Electroencephalogr Clin Neurophysiol 94:381-384.

Kitano K, Fukai T (2007) Variability vs. synchronicity of neuronal activity in local cortical network models with different wiring topologies. J Comput Neurosci 23:237-250

Kleim JA, Chan S, Pringle E, Schallert K, Procaccio V, Jimenez R, Cramer SC (2006) , BDNF val66met polymorphism is associated with modified experience-dependent plasticity in human motor cortex. Nat Neurosci 9:735-737.

Klein TA, Neumann J, Reuter M, Hennig J, von Cramon DY, Ullsperger M (2007) Genetically determined differences in learning from errors. Science 318:1642-1645.

Kolev V, Yordanova J (1997) Analysis of phase-locking is informative for studying event-related EEG activity. Biol Cybern 76:229-235.

Kolev V, Beste C, Falkenstein M, Yordanova J (2009) Error-related oscillations. Effects of aging on neural systems for behavioural monitoring. J Psychophysiol 23:216-223.

Krämer UM, Cunillera T, Càmara E, Marco-Pallarés J, Cucurell D, Nager W,
Bauer P, Schüle R, Schöls L, Rodriguez-Fornells A, Münte TF (2007) The impact of catechol-O-methyltransferase and dopamine D4 receptor genotypes on neurophysiological markers of performance monitoring. J Neurosci 27:14190-14198.

Lago-Fernández LF, Corbacho FJ, Huerta R (2001) Connection topology dependence of synchronization of neural assemblies on class 1 and 2 excitability. Neural Netw 14:687-696.

Mallat S (1999) A wavelet tour of signal processing, ed 2. San Diego: Academic.

Nunez PL, Srinivasan R, Westdorp AF, Wijesinghe RS, Tucker DM, Silberstein RB, Cadusch PJ (1997) EEG coherency. I: Statistics, reference electrode, volume conduction, Laplacians, cortical imaging, and interpretation at multiple scales. Electroencephalogr Clin Neurophysiol 103:499-515.

Oeth P, Beaulieu M, Park C, Kosman D, del Mistro G, van den Boom D, Jurinke C (2007) "iPLEX ${ }^{\mathrm{TM}}$ assay: increased plexing efficiency and flexibility for MassARRAY system through single base primer extension with mass-modified terminators." www.sequenom.com/getdoc/52ad1d40ff33-421f-a26f-58ca91b86364/iPLEX_Application_Note+Oeth+IPLEX\&hl= $\mathrm{de} \& \mathrm{gl}=$ de\&pid $=$ bl\&srcid $=$ ADGEESgYq2aCFcEpk3dtmUe8xeXG38mwpz F6Ftm7D5L61jZ980XVra_wM2q2Ugez350U35GRRM4doHnGLSDO9f1kI7zcx54rWees_HLpD1tyL6wU65jA_GxmutzVHUR3PPcjV28OqJV\&sig= AHIEtbSup8-lmgeTd0urm3pPZcLuIpeXIQ.

Perrin F, Pernier J, Bertrand O, Echallier JF (1989) Spherical splines for scalp potential and current density mapping. Eletroencephalogr Clin Neurophysiol 72:184-187.

Pezawas L, Verchinski BA, Mattay VS, Callicott JH, Kolachana BS, Straub RE, Egan MF, Meyer-Lindenberg A, Weinberger DR (2004) The brainderived neurotrophic factor val66met and variation in human cortical morphology. J Neurosci 24:10099-10102.

Rabbitt PM (1966) Error correction time without external error signals. Nature 212:438.

Roach BJ, Mathalon DH (2008) Event-related EEG time-frequency analysis: an overview of measures and an analysis of early gamma band phase locking in schizophrenia. Schizophr Bull 34:907-926.

Rybakowski JK (2008) BDNF gene: functional Val66Met polymorphism in mood disorders and schizophrenia. Pharmacogenomics 9:1589-1593.

Tallon-Baudry C, Bertrand O, Delpuech C, Permier J (1997) Oscillatory gamma-band $(30-70 \mathrm{~Hz})$ activity induced by a visual search task in humans. J Neurosci 17:722-734.

Tallon-Baudry C, Bertrand O, Fischer C (2001) Oscillatory synchrony between human extrastriate areas during visual short-term memory maintenance. J Neurosci 21:RC177.

Thomason ME, Yoo DJ, Glover GH, Gotlib IH (2009) BDNF genotype modulates resting functional connectivity in children. Front Hum Neurosci 3:55.

Varela F, Lachaux JP, Rodriguez E, Martinerie J (2001) The brainweb: phase synchronization and large-scale integration. Nat Rev Neurosci 2:229-239.

Yordanova J, Kolev V (1998) Single-sweep analysis of the theta frequency band during auditory oddball task. Psychophysiology 35:116-126.

Yordanova J, Kolev V, Hohnsbein J, Falkenstein M (2004a) Sensorimotor slowing with aging is mediated by a functional dysregulation of motorgeneration processes: evidence from high-resolution event-related potentials. Brain 127:351-362.

Yordanova J, Falkenstein M, Hohnsbein J, Kolev V (2004b) Parallel systems of error processing in the brain. Neuroimage 22:590-602. 